\title{
Bilateral Nasal Polyp with Unilateral Pathology
}

\author{
${ }^{1}$ Ramandeep Singh Virk, ${ }^{2}$ Ashok K Gupta, ${ }^{3}$ Mohnish Grover, ${ }^{3}$ Kshitij Charaya \\ ${ }^{1}$ Assistant Professor, Department of O tolaryngology and Head and Neck Surgery, Postgraduate Institute of Medical \\ Education and Research, Chandigarh, India \\ ${ }^{2}$ Professor, Department of O tolaryngology and Head and Neck Surgery, Postgraduate Institute of Medical \\ Education and Research, Chandigarh, India \\ ${ }^{3}$ S enior Resident, Department of O tolaryngology and Head and Neck Surgery, Postgraduate Institute of Medical
} Education and Research, Chandigarh, India

Correspondence: Ramandeep Singh Virk, Assistant Professor, Department of Otolaryngology and Head and Neck Surgery House No 2168, Sector 15C, Chandigarh-160015, India, Phone: +91-172-2782246, 2727178, Fax: +91-172-2771881 e-mail: virkdoc@ hotmail.com

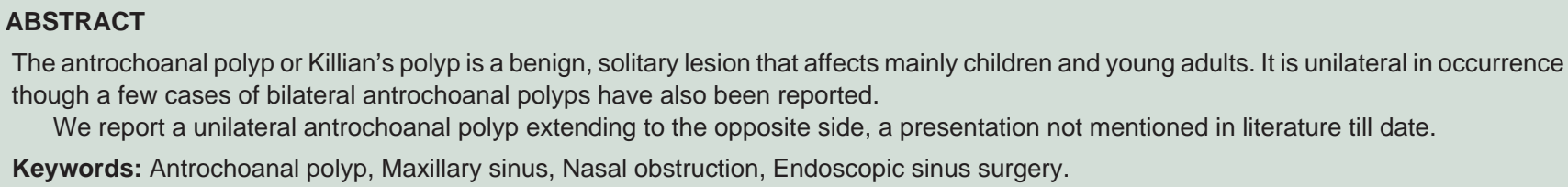

\section{INTRODUCTION}

Studies show that antrochoanal polyp accounts for 4 to $6 \%$ of all nasal polyps that affect the general population, however, in the pediatric population this percentage reaches $33 \% .{ }^{1}$ It sprouts from a hypertrophy of the maxillary sinus mucosa, near its ostium, and develops because of some unknown stimuli through the maxillary sinus ostium towards the nasal cavity and the choana, growing all the way to the nasopharynx; it may even reach the oropharynx in some instances.

The polyp shows rapid growth, probably due to the venous return of its pedicle, which is then compressed by the ostium opening, thus making the polyp increasingly more edematous. Its clinical manifestation is usually unilateral nasal obstruction, how ever, it may become bilateral in those cases in which the polyp is extremely bulky, causing nasal septum deviation. Bilateral antrochoanal polyps though known, are very rare. ${ }^{2,3}$

\section{CASE REPORT}

A 46-year-old male patient, postman by occupation, presented to us with a history of progressive nasal obstruction for last 8 months; initially right-sided, but slowly progressed to involve the left side since 2 months. He also gave history of recurrent sneezing and mucoid nasal discharge. There was no history of epistaxis, visual disturbances or postnasal drip.
On anterior rhinoscopy, right-sided nasal cavity was completely filled with a pale insensitive polyp, which did not bleed on touch. Left side examination revealed a similar polyp medial to inferior turbinate. Posterior rhinoscopy showed mass filling nasopharynx obstructing the view of the choanae.

Noncontrast-enhanced computerized tomography (NCCT) of nose and paranasal sinuses (Fig. 1) showed a soft tissue density in right maxillary antrum, bilateral nasal cavities and nasopharynx with no bony destruction. Other

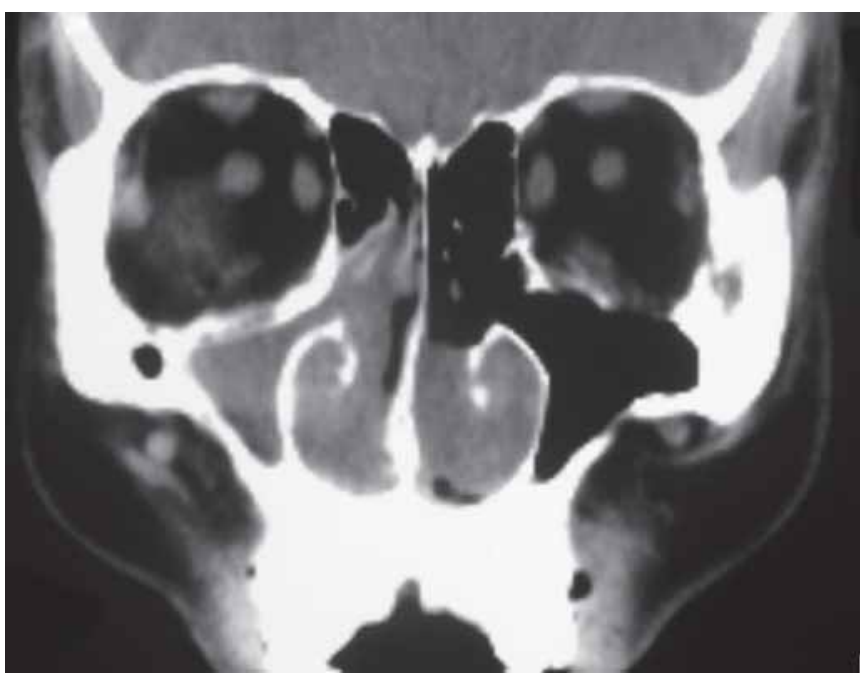

Fig. 1: CT paranasal sinuses (axial section)- soft tissue density mass involving right maxillary antrum and right nasal cavity, and left nasal cavity showing mass which could not be differentiated from the inferior turbinate 
sinuses were normal. M agnetic resonance imaging ( $\mathrm{M} \mathrm{RI}$ ) of the same region demonstrated soft tissue density in same areas with $\mathrm{T} 2$-weighted images revealing hyperintensity in right maxillary antrum, nasopharynx and left nasal cavity (Figs 2 and 3).

A diagnosis of right antrochoanal polyp and a nasal polyp in left nasal cavity, probably arising from posterior end of inferior turbinate, was made. Patient was planned for endoscopic surgery under general anesthesia. M icrodebrider (X omed M edtronicX PS3000, Jacksonville, Florida, USA ) was used to remove the right nasal polyp and then the pedicle between antral and choanal part was removed. Cystic antral part was completely removed using a $45^{\circ}$ endoscope and a curved debrider blade (RAD60 Xomed Medtronic, Jacksonville, Florida, USA). The remaining part was then pushed posteriorly into the nasopharynx and delivered via oral cavity. This brought along with it the polyp on the left side and it was seen to

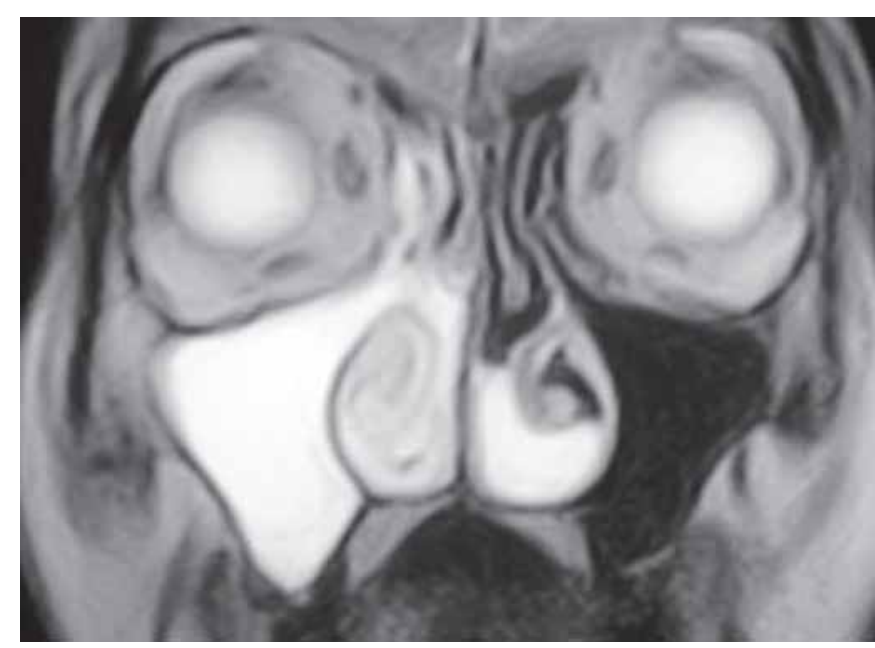

Fig. 2: MRI paranasal sinuses (axial section)-T2-weighted image showing hyperintense mass involving right maxillary antrum extending to right nasal cavity and another hyperintense mass in left nasal cavity along its floor

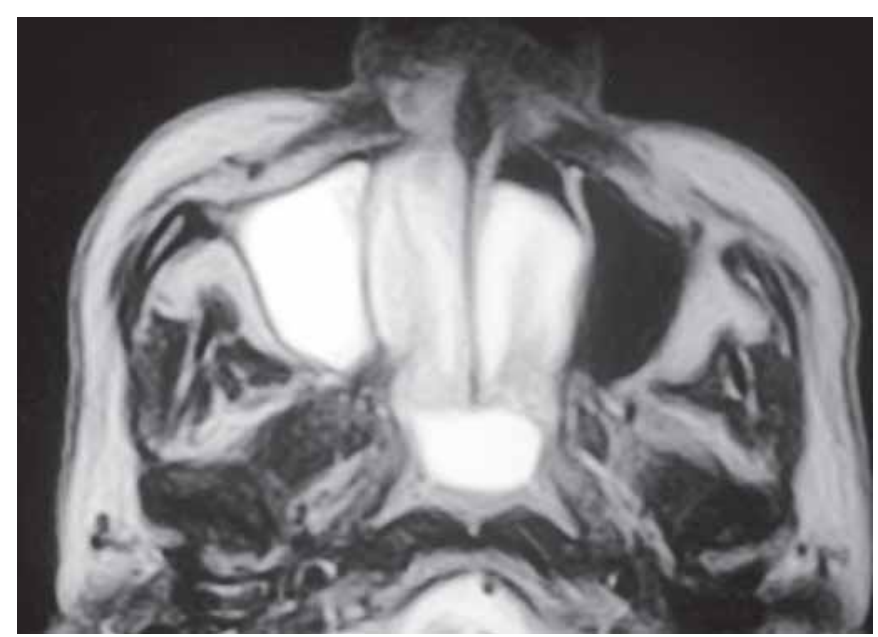

Fig. 3: MRI paranasal sinuses (coronal section)-T2-weighted image showing hyperintense lesion involving right maxillary antrum, bilateral nasal cavities and choana arise from the right antrochoanal polyp, extending into the nasopharynx and then into the left side nasal cavity. Histopathological examination showed benign inflammation.

\section{DISCUSSION}

Although this is not a rare disease, Killian's polyps have enjoyed little attention with very few cases described in the specialized literature. It is a benign, unilateral disease, which starts on the floor of the maxillary sinus, near dental roots and migrates towards the nasal cavity, cavum and oropharynx.

Its first description was made in 1753 by Palfyn, when he reported a woman with a nasopharynx polyp growing towards her uvula. Notwithstanding, it was only in 1906 that Killian, for the first time, provided a precise description of the antrochoanal polyp's natural history, stating that its origin was in the maxillary sinus and not in the posterior choana, as it was previously believed. Killian was unable to confirm its origin in the maxillary antrum, which was then done by Kubo in 1909.

The maxillary sinus ostia in the cases of antrochoanal polyps are al ways found to be large. It is unlikely to be due to expansion by the polyp, as large polyps neither cause expansion of the choana nor do they erode or displace the middle turbinate medially. Proetz suggested that the wide ostia may be a result of faulty development and may itself be the cause of antrochoanal polyp. The polyp is cystic in nature inside the antrum and as it protrudes out of maxillary ostium, all its walls come in contact with each other and then form a polypoidal mass. This mass extends anteriorly towards the anterior nares and posteriorly towards the choana and nasopharynx, where it enlarges and may cause bilateral nasal obstruction. Giant antrochoanal polyps may hang into the oropharynx and cause complete respiratory obstruction rarely, leading to emergencies. ${ }^{4}$

Patients usually present with unilateral nasal obstruction and rarely with bilateral nasal obstruction. Bilateral antrochoanal polyps are very rare. Our extensive search of the english literature revealed less than five such case reports. $^{2,3}$

We could not find even a single case report describing extension of an antrochoanal polyp into the opposite nasal cavity and thereby presenting as bilateral polyp.

The treatment options for this benign disease have also been constantly under scanner. M any surgeons are conservative in their management of antrochoanal polyp in children under eight years of age. The possibility of damage to the secondary dentition had prompted surgeons to do simple intranasal polypectomy, but recurrence was almost inevitable. Removal of antrochoanal polyp in adults by endoscopic sinus surgery has emerged as a safe and effective procedure. Even in pediatric population, most surgeons now 
prefer the use of endoscopes and the recent literature shows it to be a safe and effective modality. ${ }^{5}$

A ntrochoanal polyp or Killian's polyp is a benign nonatopic lesion of the maxillary sinus. It is important to realize that antrochoanal polyp is considered to be unilateral and is not considered in differential diagnosis in case of bilateral nasal polyps, however this case demonstrates that a unilateral antrochoanal polyp can extend to the opposite side and thereby present as bilateral nasal polyps.

The patient mentioned here was an adult male, operated by endoscopic sinus surgery with no postoperative complications and patient was relieved of bilateral nasal obstruction by performing surgery only on one side.

\section{REFERENCES}

1. Basak S, Karaman CZ, A kdilli A, M etin KK. Surgical approaches to antrochoanal polyps in children. Int J Pediatr Otorhinolaryngol 1998;46(3):197-205.

2. Basu SK, B andhyopadhyay SN, B ora H. B ilateral antrochoanal polyps. J Laryngol Otol July 2001;115:561-62.

3. M yatt $H M$, C abrera M . B ilateral antrochoanal polyps in a child: A case report. J Laryngol Otol M arch 1996;110:272-74.

4. Sharma HS, A hmad Daud AR. Antrochoanal polyp: A rare paediatric emergency. Inter J Pediatr Otorhinolaryngol; 41(1):65-70.

5. L ee TJ, Huang SF. Endoscopic sinus surgery for antrochoanal polyps in children. Otolaryngol Head Neck Surg Nov 2006; 135(5):688-92. 